\section{nature photonics}

Plasmonic collimator shapes laser output

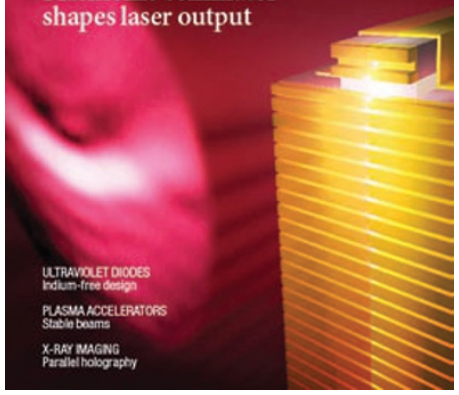

\section{Cover story}

Vol.2 No.9 September 2008

Nanfang Yu and colleagues have used surface plasmons to reduce beam divergence in semiconductor lasers. This is accomplished by patterning one facet of a quantum-cascade laser. An aperture efficiently couples the laser light into the surface-plasmon modes of a metallic grating. This grating acts like an antenna array, shaping the beam and radiating energy into the far-field. With this modification the beam divergence is reduced to just $2.4^{\circ}$, compared with $63^{\circ}$ without the aid of plasmons. What's more, this is achieved without significant decrease in the optical output power of the laser. Such collimation is important for coupling light to optical fibres and waveguides and for applications in which light is to be used over long distances in free space.

[Article p564; News \& Views p524]

\section{PENETRATING THE ULTRAVIOLET}

Electrically powered semiconductor laser diodes are a highly convenient source of laser light, but their wavelength of operation is yet to reach deep into the UV. Now, Harumasa Yoshida and co-workers report an AlGaN laser diode that operates at a wavelength of $342 \mathrm{~nm}$ - the shortest wavelength of operation reported so far. The achievement is important because there are many applications in biomedicine, data storage and materials processing that require short-wavelength UV laser diodes. The next generation of optical data-storage systems following today's Blu-ray disks may need short-wavelength UV laser diodes. In addition, semiconductor lithography for the manufacture of microchips also requires UV lasers, and fluorescence in biological systems is excited by UV light. Importantly, the laser developed by Yoshida and colleagues contains no indium. Indium is usually added to improve the efficiency of UV laser diodes, but it limits device operation to longer wavelengths. The demonstration proves that it is possible to create a practical and efficient indium-free UV laser diode that operates at room temperature. The device emits powers in the milliwatt range when driven by a pulsed electrical current. [Letter p551; News \& Views p521]

\section{X-RAY PINHOLE CAMERAS}

Pinholes have been used in imaging for centuries. Stefano Marchesini and his colleagues have now applied this concept to X-ray holography. Pinholes are very simple, but when it comes to imaging they suffer from an inconvenient trade-off. To obtain high spatial resolution the hole needs to be small, but this limits the amount of light that can get through. The way around this problem is to use more holes: each hole creates an image that can then be processed by a computer to obtain a single high-resolution picture. Marchesini et al. apply this concept to X-ray Fourier-transform holography and demonstrate its potential by imaging a bacterium. A Spiroplasma cell was placed on a silicon nitride membrane next to 162 pinholes arranged in the optimum pattern for achieving a high resolution without compromising the image brightness, a so-called binary uniformly redundant array. Using 15-fs pulses of radiation with a wavelength of $13.5 \mathrm{~nm}$, they achieved a resolution of $150 \mathrm{~nm}$, which was reduced to $75 \mathrm{~nm}$ after further processing. With the ongoing development of even shorter-wavelength light sources, this work demonstrates the very real potential for highresolution molecular movies.

[Letter p560; News \& Views p529]

\section{SCALED DOWN FREE-ELECTRON LASERS}

A new generation of free-electron lasers (FELs) aims to push high-power radiation sources to even shorter wavelengths, past the extreme UV, through the soft-X-ray region and beyond, and open up an entire realm of new science. There are a number of facilities around the world that are now

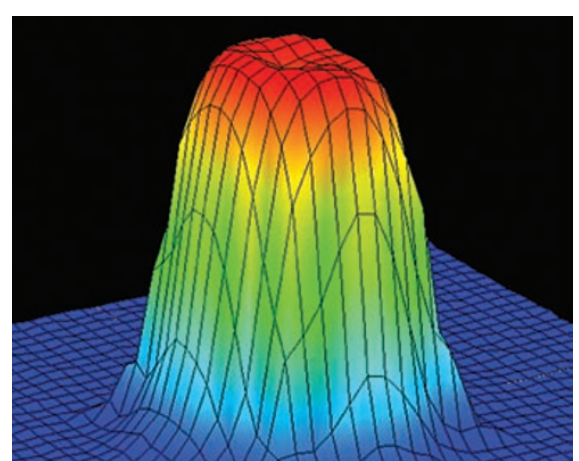

X-ray free-electron lasers are being brought down to size. A key requirement of the technology under development at the SCSS in Japan is a high-quality electron beam, which is used to kick start the generation of short-wavelength light. in development; one of these is the SPring- 8 Compact SASE (self-amplified spontaneous emission) Source (SCSS) in Japan. In this issue, scientists working on this project present the characterization of a prototype of this machine. So what makes SCSS different from its competitors? X-ray FELs are often kilometres in size. The aim of the SCSS project is to develop a technology that enables smaller machines to be made, making X-ray sources available to more researchers. The 55-m-long prototype lases at wavelengths between $51 \mathrm{~nm}$ and $61 \mathrm{~nm}$, with a maximum pulse energy of $30 \mu \mathrm{J}$. These initial results are an indication of the impressive performance that can be expected when the full SCSS comes online in 2010. [Letter p555; News \& Views p522]

\section{ENERGETIC ELECTRONS}

Laser-wakefield accelerators present the rather exciting possibility of powerful electron-beam sources that are small enough to fit on a table top. In this issue, Nasr Hafz and colleagues show how to get even more energetic electrons from these machines. A very short but intense pulse of light passing through a plasma creates a plasma wave. This wave can trap electrons and accelerate them to relativistic energies. Producing a good-quality beam depends on a lot of experimental parameters: plasma density, the size of the focused laser spot, pulse length, and much more. Hafz et al. investigate a system over a wide range of these parameters and demonstrate that it is possible to generate bunches of gigaelectronvolt electrons. For example, by focusing 35 -fs-duration laser pulses with a power of 50 TW onto a 1-cm-long helium plasma, electrons with an energy between $150 \mathrm{MeV}$ and $870 \mathrm{MeV}$ were created. In addition to the impressive generation of such high-energy electrons, the beam is also very stable and reproducible. [Article p571; News \& Views p526; Interview p580] 